Research Article

\title{
Some Existence Results for High Order Fractional Impulsive Differential Equation on Infinite Interval
}

\author{
Zhaocai Hao $\mathbb{D}^{1,2}$ and Tian Wang $\mathbb{D}^{1,3}$ \\ ${ }^{1}$ School of Mathematical Sciences, Qufu Normal University, Qufu 273165, Shandong, China \\ ${ }^{2}$ Department of Mathematics and Statistics, Missouri University of Science and Technology, Rolla, MO 65401, USA \\ ${ }^{3}$ School of Mathematics, Faculty of Science, Beijing University of Technology, Beijing 100124, China
}

Correspondence should be addressed to Tian Wang; 13562714499@163.com

Received 5 August 2020; Revised 23 September 2020; Accepted 28 September 2020; Published 22 October 2020

Academic Editor: Chuanjun Chen

Copyright (c) 2020 Zhaocai Hao and Tian Wang. This is an open access article distributed under the Creative Commons Attribution License, which permits unrestricted use, distribution, and reproduction in any medium, provided the original work is properly cited.

In this paper, we consider the high order impulsive differential equation on infinite interval $\int D_{0+}^{\alpha} u(t)+f\left(t, u(t), J_{0+}^{\beta} u(t), D_{0+}^{\alpha-1} u(t)\right)=0, t \in[0, \infty) \backslash\left\{t_{k}\right\}_{k=1}^{m}$

$\left\{\begin{array}{l}\triangle u\left(t_{k}\right)=I_{k}\left(u\left(t_{k}\right)\right), t=t_{k}, k=1, \ldots, m \\ u(0)=u^{\prime}(0)=\cdots=u^{(n-2)}(0)=0, D_{0+}^{\alpha-1} u(\infty)=u_{0}\end{array}\right.$

obtain some new results on the existence of solutions. The nonlinear term of the equation contains fractional integral operator $J^{\beta} u(t)$ and lower order derivative operator $D_{0+}^{\alpha-1} u(t)$. An example is presented to illustrate our results.

\section{Introduction}

In this paper, we are concerned with the following impulsive differential equation on infinite interval:

$$
\left\{\begin{array}{l}
D_{0+}^{\alpha} u(t)+f\left(t, u(t), J_{0+}^{\beta} u(t), D_{0+}^{\alpha-1} u(t)\right)=0, \quad t \in[0, \infty) \backslash\left\{t_{k}\right\}_{k=1}^{m}, \\
\triangle u\left(t_{k}\right)=I_{k}\left(u\left(t_{k}\right)\right), \quad t=t_{k}, k=1, \ldots, m \\
u(0)=u^{\prime}(0)=\cdots=u^{(n-2)}(0)=0, \quad D_{0+}^{\alpha-1} u(\infty)=u_{0}
\end{array}\right.
$$

where $u_{0} \in R, \alpha, \beta \in(n-1, n], n>2, D_{0+}^{\alpha}$ is the standard Riemann-Liouville fractional derivative, $0=t_{0}<t_{1}<t_{2}<\cdots<t_{m}<\infty, \quad \Delta u\left(t_{k}\right)=u\left(t_{k}^{+}\right)-u\left(t_{k}^{-}\right)$, $u\left(t_{k}^{-}\right)=u\left(t_{k}\right), \quad u\left(t_{k}^{+}\right)=\lim _{h \longrightarrow 0+} u\left(t_{k}+h\right) \quad$ and $u\left(t_{k}^{-}\right)=\lim _{h \longrightarrow 0-} u\left(t_{k}-h\right)$ represent the right and left limits of $u(t)$ at $t=t_{k}$, and $D_{0+}^{\alpha-1} u(\infty)=\lim _{t \rightarrow \infty} D_{0+}^{\alpha-1} u(t)$. Also, $f \in C([0,+\infty) \times R \times R \times R, R), I_{k} \in C(R, R)$.
During the past decades, fractional differential equations have drawn wide concerns. Compared with integer order differential equations, fractional differential equations have more extensive application range, such as control theory, physics, aerodynamics, polymer rheology, chemistry, biology, and so forth. There are many papers focused on the existence of positive solutions for fractional differential equations (see [1-3]). 
Since the last century, the dynamics of populations subject to abrupt changes was described by impulsive differential system. And other phenomena, for instance, harvesting, diseases, and so on, also have been described by using impulsive differential systems. Impulsive differential equations of fractional order play an important role in fractional differential equations theory and applications. Recently, impulsive fractional differential equations have been studied extensively. For example, Wang et al. studied the existence and multiplicity of solutions for impulsive fractional boundary value problem with p-Laplacian in [4], and Liu considered fractional impulsive differential equations using bifurcation techniques in [5]. For more articles related to impulsive fractional differential equations, refer to [6-12].

Recently, in [13], Liu investigated the existence of solutions for higher order impulsive fractional differential equations given by

$$
\begin{aligned}
& \left\{\begin{array}{l}
{ }^{c} D_{0+}^{q} x(t)=F(t, x(t)), \quad t \in\left(t_{i}, t_{i+1}\right], i \in N_{0}, \\
\left.\triangle x\right|_{t=t_{i}}=I\left(t_{i}, x\left(t_{i}\right)\right), \quad i \in N, \\
x(0)=x_{0},
\end{array}\right. \\
& \left\{\begin{array}{l}
D_{0+}^{q} x(t)=G(t, x(t)), \quad t \in\left(t_{i}, t_{i+1}\right], i \in N_{0}, \\
\lim _{t \longrightarrow t_{i}^{+}}\left(t-t_{i}^{1-\alpha}\right) x(t)=J\left(t_{i}, x\left(t_{i}\right)\right), \quad i \in N, \\
\lim _{t \longrightarrow 0^{+}} t^{1-q} x(t)=x_{0},
\end{array}\right.
\end{aligned}
$$

where $\quad q \in(0,1), \quad t \in[0, T], \quad 0=t_{0}<t_{1}<t_{2}<\cdots$ $<t_{m}<t_{m+1}<T, \quad I, J:\left\{t_{k}: k \in N\right\} \times R \longrightarrow R$ are discrete Carathéodory functions, and $F, G:(0, T) \times R \longrightarrow R$ are strong Carathéodory functions. By using Schauder's fixedpoint theorem, Liu established some existence results.

In [10], Liu and Ahmad studied the following problems:

$$
\begin{aligned}
& \left\{\begin{array}{l}
{ }^{c} D_{0+}^{\alpha} x(t)=q(t) f\left(t, x(t),{ }^{c} D_{0+}^{p} x(t)\right), \quad t \in(0, \infty), \\
\triangle x\left(t_{k}\right)=I_{k}\left(t_{k}, x\left(t_{k}\right)\right), \quad k=1,2, \ldots, \\
x(0)=x_{0},
\end{array}\right. \\
& \left\{\begin{array}{l}
{ }^{c} D^{\alpha} x(t)=q(t) f\left(t, x(t),{ }^{c} D_{*}^{p} x(t)\right), \quad t \in(0, \infty), \\
\triangle x\left(t_{k}\right)=I_{k}\left(t_{k}, x\left(t_{k}\right)\right), \quad k=1,2, \ldots, \\
x(0)=x_{0},
\end{array}\right.
\end{aligned}
$$

where $x_{0} \in R, \alpha \in(0,1], 0<p<\alpha, 0=t_{0}<t_{1}<t_{2}<\cdots$ with $\lim _{k \longrightarrow \infty} t_{k}=\infty, q:(0, \infty) \longrightarrow R$ satisfies that there exists $l>-\alpha$ such that $|q(t)| \leq t^{l}$ for all $t \in(0, \infty)$, and $q$ may be singular at $t=0$. And $f:[0, \infty) \times R^{2} \longrightarrow R$ is a Carathéodory function, $I_{k}:(0, \infty) \times R \longrightarrow R(k=1,2, \ldots),, I_{k}$ is a Carathéodory function sequence, and $\triangle x\left(t_{k}\right)=\lim _{t \longrightarrow t_{k}^{+}} x(t)-\lim _{t \longrightarrow t_{k}^{-}} x(t), k=1,2, \ldots$, By using Schauder's fixed-point theorem, the authors studied the existence of solution. And the authors also considered the uniqueness of solution under some appropriate conditions.
In [9], Zhao and Ge considered the following boundary value problem:

$$
\left\{\begin{array}{l}
D_{0+}^{\alpha} u(t)+f(t, u(t))=0, \quad t \in(0, \infty), t \neq t_{k}, k=1,2, \ldots, m, \\
u\left(t_{k}^{+}\right)-u\left(t_{k}^{-}\right)=-I_{k}\left(u\left(t_{k}\right)\right), \quad k=1,2, \ldots, m, \\
u(0)=0, \quad D_{0+}^{\alpha} u(\infty)=0,
\end{array}\right.
$$

where $\alpha$ is a real number with $1<\alpha \leq 2, D_{0+}^{\alpha}$ is the standard Riemann-Liouville fractional derivative, $t_{0}=0$, $1<t_{1}<t_{2}<\cdots<t_{m}<\infty, \quad u\left(t_{k}^{+}\right)=\lim _{h \longrightarrow 0^{+}} u\left(t_{k}+h\right)$, $u\left(t_{k}^{-}\right)=\lim _{h \longrightarrow 0^{+}} u\left(t_{k}-h\right), D_{0+}^{\alpha-1} u(\infty)=\lim _{t \rightarrow \infty} D_{0+}^{\alpha-1} u(t)$, $f\left(t,\left(1+t^{\alpha}\right) u\right):[0, \infty) \times[0, \infty) \longrightarrow[0, \infty)$ is continuous, and $I_{k}:[0, \infty) \longrightarrow[0, \infty)(k=1,2, \ldots, m)$ are continuous. Wang and Ge proved that the problem they studied has at least three positive solutions.

Motivated by the aforementioned work, we studied existence of solution of problem (1) by Schauder's fixedpoint theorem and Altman's fixed-point theorem. The main features of this paper are as follows. Firstly, the nonlinear term not only involved fractional order derivative but also contained fractional integral. Compared with $[9,10,13]$, our nonlinear terms are more general. Many articles contain derivatives for nonlinear terms, but few articles contain both derivatives and integrals. Secondly, we studied the problem on the infinite interval. To the best of our knowledge, there are few articles involving the impulsive fractional order differential equations on the infinite interval. If the nonlinear term contained fractional integral and $t \in[0, \infty)$, it will bring new obstacles to solve the problem. For this purpose, we overcome obstacles by constructing a special cone. Thirdly, our problem is higher order impulsive fractional equation. Compared with [9], we allowed $\alpha \in(n-1, n]$, where $n>2$. It is obvious that our problem is more general.

This paper is organized as follows. In Section 2, we introduce some definitions and lemmas. In Section 3, we give our main results by fixed-point theorem. In Section 4, one example is presented to illustrate the main results.

\section{Preliminaries and Lemmas}

Let $u:[0, \infty) \longrightarrow \mathbb{R}, J=[0, \infty), J_{0}=\left[0, t_{1}\right], J_{m}=\left(t_{m}, \infty\right)$, $J_{k}=\left(t_{k}, t_{k+1}\right], k=1, \ldots, m-1$. For $k=1,2, \ldots, m$, define the function $u_{k}(t)=u(t)$. Let $C(J, R)$ be the Banach space of continuous functions from $J$ to $\mathbb{R}$. Let us to introduce the Banach spaces

$$
\begin{aligned}
\operatorname{PC}(J, \mathbb{R})= & \left\{u: u_{k} \in C\left(J_{k}, \mathbb{R}\right), k=0,1, \ldots, m, u\right. \\
& \cdot\left(t_{k}^{+}\right) \text {and } u\left(t_{k}^{-}\right) \text {exist, } u\left(t_{k}\right) \\
= & \left.u\left(t_{k}^{-}\right), \lim _{t \rightarrow \infty} \frac{u(t)}{1+t^{\alpha-1}} \text { exsits }\right\},
\end{aligned}
$$

with the norm 


$$
\begin{aligned}
\|u\|_{\mathrm{PC}} & =\sup _{t \in[0, \infty)}\left|\frac{u(t)}{1+t^{\alpha-1}}\right| \\
\operatorname{PC}^{1}(J, \mathbb{R}) & =\left\{u \in \mathrm{PC}(J, R): D^{\alpha-1} u(t) \in C\left(J_{k}, \mathbb{R}\right), k=0,1, \ldots, m, D^{\alpha-1} u\left(t_{k}^{+}\right) \text {and } D^{\alpha-1} u\left(t_{k}^{-}\right) \text {exist, } D^{\alpha-1} u\left(t_{k}^{-}\right)\right. \\
& \left.=D^{\alpha-1} u\left(t_{k}\right), \lim _{t \rightarrow \infty} D^{\alpha-1} u(t) \text { exists }\right\},
\end{aligned}
$$

with the norm

$$
\|u\|_{\mathrm{PC}^{1}}=\max \left\{\sup _{t \in J} \frac{|u(t)|}{1+t^{\alpha-1}}, \sup _{t \in J}\left|D^{\alpha-1} u(t)\right|\right\} .
$$

Definition 1. The Riemann-Liouville fractional integral of order $\alpha>0$ of a function $f:(0, \infty) \longrightarrow \mathbb{R}$ is given by

$$
J_{0+}^{\alpha} f(t)=\frac{1}{\Gamma(\alpha)} \int_{0}^{t}(t-s)^{\alpha-1} f(s) \mathrm{d} s,
$$

where the right side is pointwise defined on $(0, \infty)$.

Definition 2. The Riemann-Liouville fractional derivative of order $\alpha>0$ of a function $f:(0, \infty) \longrightarrow \mathbb{R}$ is given by

$$
D_{0+}^{\alpha} f(t)=\frac{1}{\Gamma(n-\alpha)}\left(\frac{\mathrm{d}}{\mathrm{d} t}\right)^{n} \int_{0}^{t}(t-s)^{n-\alpha-1} f(s) \mathrm{d} s,
$$

where $n$ is the smallest integer greater than or equal to $\alpha$ and the right side is pointwise defined on $(0, \infty)$. In particular, for $\alpha=n, D_{0+}^{\alpha} f(t)=f^{(n)}(t)$.

Lemma 1. Let $\alpha>0$, and $n$ denotes the smallest integer greater than or equal to $\alpha$. For all $t \in[a, b]$,

$$
J_{0+}^{\alpha} D_{0+}^{\alpha} u(t)=u(t)+c_{1} t^{\alpha-1}+c_{2} t^{\alpha-2}+\cdots+c_{n} t^{\alpha-n},
$$

where $c_{j} \in \mathbb{R}, j=1,2, \ldots, n$.

Lemma 2 (see [2]). Let $\Omega \subseteq P C^{1}$. Then, $\Omega$ is relatively compact in $P C^{1}$ if the following conditions hold:

(1) $\Omega$ is bounded in $P C^{1}$

(2) For any $u(t) \in \Omega, u(t) / 1+t^{\alpha-1}$ and $D^{\alpha-1} u(t)$ are equicontinuous on any interval $J_{k}$

(3) Given $\varepsilon>0$, there exists a constant $N=N(\varepsilon)>0$ such that

$$
\begin{array}{r}
\left|\frac{u\left(t_{1}\right)}{1+t_{1}^{\alpha-1}}-\frac{u\left(t_{2}\right)}{1+t_{2}^{\alpha-1}}\right|<\varepsilon, \\
\left|D^{\alpha-1} u\left(t_{1}\right)-D^{\alpha-1} u\left(t_{2}\right)\right|<\varepsilon,
\end{array}
$$

for any $t_{1}, t_{2} \geq N$ and $u(t) \in \Omega$.

Theorem 1 (Schauder fixed-point theorem). If $U$ is a closed bounded convex subset of a Banach space $X$ and
$T: U \longrightarrow U$ is completely continuous, then $T$ has at least one fixed point in $U$.

Theorem 2 (Altman theorem). Let $\Omega$ be an open bounded subset of a Banach space $E$ with $0 \in \Omega$ and $T: \bar{\Omega} \longrightarrow E$ be a completely continuous operator. Then, $T$ has a fixed point in $\bar{\Omega}$, provided that

$$
\|\mathrm{Tx}-x\|^{2} \geq\|\mathrm{Tx}\|^{2}-\|x\|^{2}, \quad \forall x \in \partial \Omega .
$$

Lemma 3. For a given $y \in C(J, \mathbb{R})$, a function $u \in P C^{1}(J, \mathbb{R})$ is a solution of the following boundary value problem:

$$
\left\{\begin{array}{l}
D_{0+}^{\alpha} u(t)+y(t)=0, \quad t \in[0, \infty) \backslash\left\{t_{k}\right\}_{k=1}^{m}, \\
\triangle u\left(t_{k}\right)=I_{k}\left(u\left(t_{k}\right)\right), \quad t=t_{k}, \\
u(0)=u^{\prime}(0)=\cdots=u^{(n-2)}(0)=0, \quad D_{0+}^{\alpha-1} u(\infty)=u_{0},
\end{array}\right.
$$

if and only if $u \in P C^{1}(J, \mathbb{R})$ is a solution of the impulsive fractional integral equation

$$
\begin{aligned}
u(t)= & -\frac{1}{\Gamma(\alpha)} \int_{0}^{t}(t-s)^{\alpha-1} y(s) \mathrm{d} s+\frac{t^{\alpha-1}}{\Gamma(\alpha)} \int_{0}^{\infty} y(s) \mathrm{d} s \\
& +\frac{t^{\alpha-1}}{\Gamma(\alpha)} u_{0}-t^{\alpha-1} \sum_{t<t_{i}} I_{i} t_{i}^{1-\alpha} .
\end{aligned}
$$

Proof. Assume $u(t)$ satisfies (13). We denote the solution of (13) by $u(t) \triangleq u_{k}(t)$ in $J_{k}(k=0,1, \ldots, m)$.

For $t \in\left[0, t_{1}\right]$, applying Lemma 1 , we have

$$
\begin{aligned}
u_{0}(t)= & -\frac{1}{\Gamma(\alpha)} \int_{0}^{t}(t-s)^{\alpha-1} y(s) \mathrm{d} s+C_{01} t^{\alpha-1}+C_{02} t^{\alpha-2} \\
& +\cdots+C_{0 n} t^{\alpha-n} .
\end{aligned}
$$

From $u(0)=u^{\prime}(0)=\cdots=u^{(n-2)}(0)=0$, we know $C_{0 n}=\cdots=C_{03}=C_{02}=0$. So, we get

$$
\begin{aligned}
& u_{0}(t)=-\frac{1}{\Gamma(\alpha)} \int_{0}^{t}(t-s)^{\alpha-1} y(s) \mathrm{d} s+C_{01} t^{\alpha-1}, \quad t \in\left[0, t_{1}\right] \\
& u\left(t_{1}^{-}\right)=-\frac{1}{\Gamma(\alpha)} \int_{0}^{t_{1}}\left(t_{1}-s\right)^{\alpha-1} y(s) \mathrm{d} s+C_{01} t_{1}^{\alpha-1} .
\end{aligned}
$$

For $t \in\left(t_{1}, t_{2}\right]$, by applying Lemma 1 , we know 


$$
\begin{aligned}
u_{1}(t)= & -\frac{1}{\Gamma(\alpha)} \int_{0}^{t}(t-s)^{\alpha-1} h(s) \mathrm{d} s+C_{11} t^{\alpha-1}+C_{12} t^{\alpha-2} \\
& +\cdots+C_{1 n} t^{\alpha-n} .
\end{aligned}
$$

In view of $u(0)=u^{\prime}(0)=\cdots=u^{(n-2)}(0)=0$, we have $C_{1 n}=\cdots=C_{13}=C_{12}=0$. So, we know

$$
\begin{aligned}
& u_{1}(t)=-\frac{1}{\Gamma(\alpha)} \int_{0}^{t}(t-s)^{\alpha-1} y(s) \mathrm{d} s+C_{11} t^{\alpha-1}, \\
& u\left(t_{1}^{+}\right)=-\frac{1}{\Gamma(\alpha)} \int_{0}^{t_{1}}\left(t_{1}-s\right)^{\alpha-1} y(s) \mathrm{d} s+C_{11} t_{1}^{\alpha-1} .
\end{aligned}
$$

And from impulsive condition of (13), $\triangle u\left(t_{1}\right)=u\left(t_{1}^{+}\right)-u\left(t_{1}^{-}\right)=I_{1}\left(u\left(t_{1}\right)\right)$. Then,

$$
\begin{aligned}
& -\frac{1}{\Gamma(\alpha)} \int_{0}^{t_{1}}\left(t_{1}-s\right)^{\alpha-1} y(s) \mathrm{d} s+C_{11} t_{1}^{\alpha-1} \\
& -\left(-\frac{1}{\Gamma(\alpha)} \int_{0}^{t_{1}}\left(t_{1}-s\right)^{\alpha-1} y(s) \mathrm{d} s+C_{01} t_{1}^{\alpha-1}\right)=I_{1}\left(u\left(t_{1}\right)\right) .
\end{aligned}
$$

Thus,

$$
C_{11}=C_{01}+t_{1}^{1-\alpha} I_{1}\left(u\left(t_{1}\right)\right)
$$

Then,

$$
\begin{aligned}
u_{1}(t)= & -\frac{1}{\Gamma(\alpha)} \int_{0}^{t}(t-s)^{\alpha-1} y(s) \mathrm{d} s+t^{\alpha-1} C_{01} \\
& +t^{\alpha-1} t_{1}^{1-\alpha} I_{1}\left(u\left(t_{1}\right)\right), \quad t \in\left(t_{1}, t_{2}\right] .
\end{aligned}
$$

For $t \in\left(t_{2}, t_{3}\right]$, by applying Lemma 1 , we obtain

$$
\begin{aligned}
u_{2}(t)= & -\frac{1}{\Gamma(\alpha)} \int_{0}^{t}(t-s)^{\alpha-1} h(s) \mathrm{d} s+C_{21} t^{\alpha-1}+C_{22} t^{\alpha-2} \\
& +\cdots+C_{2 n} t^{\alpha-n} .
\end{aligned}
$$

In view of $u(0)=u^{\prime}(0)=\cdots=u^{(n-2)}(0)=0$, we have $C_{2 n}=\cdots=C_{23}=C_{22}=0$. So, we know

$$
\begin{aligned}
& u_{2}(t)=-\frac{1}{\Gamma(\alpha)} \int_{0}^{t}(t-s)^{\alpha-1} y(s) \mathrm{d} s+C_{21} t^{\alpha-1}, \\
& u\left(t_{2}^{+}\right)=-\frac{1}{\Gamma(\alpha)} \int_{0}^{t_{2}}\left(t_{2}-s\right)^{\alpha-1} y(s) \mathrm{d} s+C_{21} t_{2}^{\alpha-1} .
\end{aligned}
$$

$\begin{array}{lcc}\text { And } & \text { from } & \text { impulsive } \\ \left(t_{2}\right)=u\left(t_{2}^{+}\right)-u\left(t_{2}^{-}\right)=I_{2}\left(u\left(t_{2}\right)\right) \text {. Then }\end{array}$

$$
\begin{aligned}
- & \frac{1}{\Gamma(\alpha)} \int_{0}^{t_{2}}\left(t_{2}-s\right)^{\alpha-1} y(s) \mathrm{d} s+C_{21} t_{2}^{\alpha-1} \\
& -\left(-\frac{1}{\Gamma(\alpha)} \int_{0}^{t_{2}}\left(t_{2}-s\right)^{\alpha-1} y(s) \mathrm{d} s+C_{11} t_{2}^{\alpha-1}\right)=I_{2}\left(u\left(t_{2}\right)\right) .
\end{aligned}
$$

We get

$$
\begin{aligned}
C_{21}= & C_{11}+t_{2}^{1-\alpha} I_{2}\left(u\left(t_{2}\right)\right)=C_{01}+t_{1}^{1-\alpha} I_{1}\left(u\left(t_{1}\right)\right) \\
& +t_{2}^{1-\alpha} I_{2}\left(u\left(t_{2}\right)\right)=C_{01}+\sum_{i=1}^{2} t_{i}^{1-\alpha} I_{i} .
\end{aligned}
$$

Consequently,

$$
\begin{aligned}
u_{2}(t)= & -\frac{1}{\Gamma(\alpha)} \int_{0}^{t}(t-s)^{\alpha-1} y(s) \mathrm{d} s+t^{\alpha-1} C_{01} \\
& +t^{\alpha-1} \sum_{i=1}^{2} t_{i}^{1-\alpha} I_{i}, \quad t \in\left(t_{2}, t_{3}\right] .
\end{aligned}
$$

By the recurrent method and Lemma 1 , for $t \in\left(t_{k}, t_{k+1}\right]$, $k=0,1,2, \ldots, m$, we can say that

$$
u(t) u_{k}(t)=-\frac{1}{\Gamma(\alpha)} \int_{0}^{t}(t-s)^{\alpha-1} y(s) \mathrm{d} s+t^{\alpha-1} C_{01}+t^{\alpha-1} \sum_{i=1}^{k} t_{i}^{1-\alpha} I_{i} .
$$

Thus, for $t \in\left(t_{m}, \infty\right)$, we have

$$
u(t)=u_{m}(t)=-\frac{1}{\Gamma(\alpha)} \int_{0}^{t}(t-s)^{\alpha-1} y(s) \mathrm{d} s+t^{\alpha-1} C_{01}
$$

$$
+t^{\alpha-1} \sum_{i=1}^{m} t_{i}^{1-\alpha} I_{i}
$$

From $D_{0+}^{\alpha-1} u(\infty)=u_{0}$, we get

$$
-\int_{0}^{\infty} y(s) \mathrm{d} s+\Gamma(\alpha) \sum_{i=1}^{m} t_{i}^{1-\alpha} I_{i}+\Gamma(\alpha) C_{01}=u_{0} .
$$

So,

$$
C_{01}=\frac{1}{\Gamma(\alpha)} u_{0}+\frac{1}{\Gamma(\alpha)} \int_{0}^{\infty} y(s) \mathrm{d} s-\sum_{i=1}^{m} t_{i}^{1-\alpha} I_{i} .
$$

Therefore, for $t \in[0, \infty)$, we have

$$
\begin{aligned}
u(t)= & -\frac{1}{\Gamma(\alpha)} \int_{0}^{t}(t-s)^{\alpha-1} y(s) \mathrm{d} s+\frac{t^{\alpha-1}}{\Gamma(\alpha)} \int_{0}^{\infty} y(s) \mathrm{d} s \\
& +\frac{t^{\alpha-1}}{\Gamma(\alpha)} u_{0}-t^{\alpha-1} \sum_{i=1}^{m} t_{i}^{1-\alpha} I_{i}+t^{\alpha-1} \sum_{t_{i}<t} t_{i}^{1-\alpha} I_{i} \\
= & -\frac{1}{\Gamma(\alpha)} \int_{0}^{t}(t-s)^{\alpha-1} y(s) \mathrm{d} s+\frac{t^{\alpha-1}}{\Gamma(\alpha)} \int_{0}^{\infty} y(s) \mathrm{d} s \\
& +\frac{t^{\alpha-1}}{\Gamma(\alpha)} u_{0}-t^{\alpha-1} \sum_{t<t_{i}} t_{i}^{1-\alpha} I_{i} .
\end{aligned}
$$

Conversely, assume that $u(t)$ satisfies impulsive fractional integral equation (14). Obviously, we get $u(0)=u^{\prime}(0)=\cdots=u^{n-2}(0)=0$, and $D_{0+}^{\alpha-1} u(\infty)=u_{0}$. Using the fact $D_{0+}^{\alpha} t^{\alpha-1}=0$, we obtain $D_{0+}^{\alpha} u(t)=-y(t)$. Also, we can easily show that $\Delta u\left(t_{k}\right)=I_{k}\left(u\left(t_{k}\right)\right), k=1,2, \ldots, m$. Then, $u$ is also the solution of problem (13). 


\section{Main Results}

In this section, we will prove the existence of solution of (1) by using Schauder fixed-point theorem and Altman theorem. first.

According to Lemma 3, we obtain the following lemma

Lemma 4. $u \in P C^{1}(J, \mathbb{R})$ is a solution of problem (1) if and only if $u \in P C^{1}(J, \mathbb{R})$ is a solution of the impulsive fractional integral equation

$$
\begin{aligned}
u(t)= & -\frac{1}{\Gamma(\alpha)} \int_{0}^{t}(t-s)^{\alpha-1} f\left(s, u(s), J^{\beta} u(s), D^{\alpha-1} u(s)\right) \mathrm{d} s \\
& +\frac{t^{\alpha-1}}{\Gamma(\alpha)} \int_{0}^{\infty} f\left(s, u(s), J^{\beta} u(s), D^{\alpha-1} u(s)\right) \mathrm{d} s \\
& +\frac{t^{\alpha-1}}{\Gamma(\alpha)} u_{0}-t^{\alpha-1} \sum_{t<t_{i}} I_{i} t_{i}^{1-\alpha}, \quad t \in J .
\end{aligned}
$$

Define an operator $T: \operatorname{PC}^{1}(J, \mathbb{R}) \longrightarrow \mathrm{PC}^{1}(J, \mathbb{R})$ as follows:

$$
\begin{aligned}
(\mathrm{Tu})(t)= & -\frac{1}{\Gamma(\alpha)} \int_{0}^{t}(t-s)^{\alpha-1} f\left(s, u(s), J^{\beta} u(s), D^{\alpha-1} u(s)\right) \mathrm{d} s \\
& +\frac{t^{\alpha-1}}{\Gamma(\alpha)} \int_{0}^{\infty} f\left(s, u(s), J^{\beta} u(s), D^{\alpha-1} u(s)\right) \mathrm{d} s \\
& +\frac{t^{\alpha-1}}{\Gamma(\alpha)} u_{0}-t^{\alpha-1} \sum_{t<t_{i}} I_{i} t_{i}^{1-\alpha}, \quad t \in J .
\end{aligned}
$$

Then, problem (1) has a solution if and only if the operator $T$ has a fixed point.

Theorem 3. Assume that following conditions hold:

(H1) For $f \in C([0,+\infty)) \times R \times R \times R, R)$, there exist nonnegative functions $a(t), b(t), c(t), e(t) \in L^{1}(J)$ such that

$$
|f(t, x, y, z)| \leq a(t)|x|+b(t)|y|+e(t)|z|+c(t),
$$

$$
\begin{aligned}
& \int_{0}^{+\infty}\left(\left(1+t^{\alpha-1}\right) a(t)+b(t)\right) \mathrm{d} t<\infty, \\
& \qquad \int_{0}^{+\infty} c(t) \mathrm{d} t<\infty, \int_{0}^{+\infty} \frac{(1+t)^{\alpha-1} t^{\beta}}{\Gamma(\beta+1)} e(t) \mathrm{d} t<\infty .
\end{aligned}
$$

(H2) For $I_{k} \in C(R, R)$, for all $u \in \mathbb{R}$, there exist some constants $L_{k}>0$ such that $\left|I_{k}(u)\right|<L_{k}, k=1,2, \ldots, m$.

Then, problem (1) has at least one solution $u(t)$ in $\operatorname{PC}^{l}(J, \mathbb{R})$.

Proof. We will use five steps to prove our conclusion. Firstly, we will show $T: \operatorname{PC}^{1}(J, \mathbb{R}) \longrightarrow \operatorname{PC}^{1}(J, \mathbb{R})$ is continuous. From (33), we know

$$
D^{\alpha-1} T u(t)=-\int_{0}^{t} f\left(s, u(s), J^{\beta} u(s), D^{\alpha-1} u(s)\right) \mathrm{d} s+\int_{0}^{\infty} f\left(s, u(s), J^{\beta} u(s), D^{\alpha-1} u(s)\right) \mathrm{d} s+u_{0}-\Gamma(\alpha) \sum_{t<t_{i}} I_{i} t_{i}^{1-\alpha} .
$$

From $(H 1)$, we have

$$
\begin{aligned}
\int_{0}^{\infty}\left|f\left(s, u(s), J^{\beta} u(s), D^{\alpha-1} u(s)\right)\right| \mathrm{d} s & \left.\leq \int_{0}^{+\infty}\left[a(s)|u(s)|+b(s) \mid D^{\alpha-1} u(s)\right)|+e(s)| J^{\beta} u(s) \mid+c(s)\right] \mathrm{d} s \\
& \left.\leq \int_{0}^{+\infty}\left[\left(1+s^{\alpha-1}\right) a(s)\|u\|_{\mathrm{PC}}+b(s) \mid D^{\alpha-1} u(s)\right) \mid+\frac{(1+s)^{\alpha-1} s^{\beta}}{\Gamma(\beta+1)} e(s)\|u\|_{\mathrm{PC}}+c(s)\right] \mathrm{d} s \\
& \leq\|u\|_{\mathrm{PC}^{1}} \int_{0}^{+\infty}\left[\left(1+s^{\alpha-1}\right) a(s)+b(s)+\frac{(1+s)^{\alpha-1} s^{\beta}}{\Gamma(\beta+1)} e(s)\right] \mathrm{d} s+\int_{0}^{+\infty} c(s) \mathrm{d} s \\
& <\infty .
\end{aligned}
$$

Let $u_{n}, u \in \mathrm{PC}^{1}(J, \mathbb{R})$ be such that $u_{n} \longrightarrow u(n \longrightarrow \infty)$. Then, $\left\|u_{n}\right\|_{\mathrm{PC}^{1}}<\infty$ and $\|u\|_{\mathrm{PC}^{1}}<\infty$. By (36) and the Lebesgue dominated convergence theorem, we get

$$
\begin{gathered}
\lim _{n \longrightarrow \infty} \int_{0}^{\infty} f\left(s, u_{n}(s), J^{\beta} u_{n}(s), D^{\alpha-1} u_{n}(s)\right) \mathrm{d} s \\
=\int_{0}^{\infty} f\left(s, u(s), J^{\beta} u(s), D^{\alpha-1} u(s)\right) \mathrm{d} s .
\end{gathered}
$$


By (H1), (H2), and (36), we have

$$
\begin{aligned}
\left|\frac{T u(t)}{1+t^{\alpha-1}}\right|= & \mid-\frac{1}{\Gamma(\alpha)} \int_{0}^{t} \frac{(t-s)^{\alpha-1}}{1+t^{\alpha-1}} f\left(s, u(s), J^{\beta} u(s), D^{\alpha-1} u(s)\right) \mathrm{d} s+\frac{t^{\alpha-1}}{1+t^{\alpha-1}} \frac{1}{\Gamma(\alpha)} \int_{0}^{\infty} f\left(s, u(s), J^{\beta} u\right. \\
& \left.\cdot(s), D^{\alpha-1} u(s)\right) \mathrm{d} s+\frac{t^{\alpha-1}}{1+t^{\alpha-1}} \frac{1}{\Gamma(\alpha)} u_{0}-\frac{t^{\alpha-1}}{1+t^{\alpha-1}} \sum_{t<t_{i}} I_{i} t_{i}^{1-\alpha} \mid \\
\leq & \frac{2}{\Gamma(\alpha)} \int_{0}^{\infty}\left|f\left(s, u(s), J^{\beta} u(s), D^{\alpha-1} u(s)\right)\right| \mathrm{d} s+\frac{\left|u_{0}\right|}{\Gamma(\alpha)}+\sum_{t<t_{i}} L_{i} t_{i}^{1-\alpha}<\infty, \\
\left|D^{\alpha-1} \mathrm{Tu}(t)\right|= & \left|-\int_{0}^{t} f\left(s, u(s), J^{\beta} u(s), D^{\alpha-1} u(s)\right) \mathrm{d} s+\int_{0}^{\infty} f\left(s, u(s), J^{\beta} u(s), D^{\alpha-1} u(s)\right)+u_{0}-\Gamma(\alpha) \sum_{t<t_{i}} I_{i} t_{i}^{1-\alpha}\right| \\
\leq & \int_{0}^{\infty}\left|f\left(s, u(s), J^{\beta} u(s), D^{\alpha-1} u(s)\right)\right| \mathrm{d} s+\int_{0}^{\infty}\left|f\left(s, u(s), J^{\beta} u(s), D^{\alpha-1} u(s)\right)\right| \mathrm{d} s+\left|u_{0}\right|+\Gamma(\alpha) \sum_{t<t_{i}} L_{i} t_{i}^{1-\alpha}<\infty .
\end{aligned}
$$

Hence, according to (37)-(39) and Lebesgue dominated convergence theorem, we can easily get

$$
\left\|\mathrm{Tu}_{n}-\mathrm{Tu}\right\|_{\mathrm{PC}^{1}} \longrightarrow 0 \quad(n \longrightarrow \infty) \text {. }
$$

Therefore, $T: \mathrm{PC}^{1}(J, \mathbb{R}) \longrightarrow \mathrm{PC}^{1}(J, \mathbb{R})$ is continuous. Secondly, choose $r$ such that

$$
r \geq \frac{2 \int_{0}^{\infty} c(s) \mathrm{d} s+\left|u_{0}\right|+\Gamma(\alpha) \sum_{t<t_{i}} L_{i} t_{i}^{1-\alpha}}{1-2 \int_{0}^{\infty}\left(\left(1+s^{\alpha-1}\right) a(s)+b(s)+(1+s)^{\alpha-1} s^{\beta} / \Gamma(\beta+1) e(s)\right) \mathrm{d} s},
$$

and let $B_{r}=\left\{\left\|u \in \mathrm{PC}^{1}\right\| u \|_{P C^{1}} \leq r\right\} \subset \mathrm{PC}^{1}(J, \mathbb{R})$.

For any $u(t) \in B_{r}$, by (41) and condition (H1), we have

$$
\begin{aligned}
& \left|\frac{\mathrm{Tu}(t)}{1+t^{\alpha-1}}\right| \leq \frac{2}{\Gamma(\alpha)} \int_{0}^{\infty}\left|f\left(s, u(s), J^{\beta} u(s), D^{\alpha-1} u(s)\right)\right| \mathrm{d} s+\frac{\left|u_{0}\right|}{\Gamma(\alpha)}+\sum_{t<t_{i}} L_{i} t_{i}^{1-\alpha} \\
& \leq \frac{2\|u\|_{\mathrm{PC}}}{\Gamma(\alpha)} \int_{0}^{+\infty}\left[\left(1+s^{\alpha-1}\right) a(s)+b(s)\right] \mathrm{d} s+\frac{2}{\Gamma(\alpha)} \int_{0}^{+\infty} c(s) \mathrm{d} s \\
& +\frac{2\|u\|_{\mathrm{PC}^{1}}}{\Gamma(\alpha)} \int_{0}^{+\infty} \frac{(1+s)^{\alpha-1} s^{\beta}}{\Gamma(\beta+1)} e(s) \mathrm{d} s+\frac{\left|u_{0}\right|}{\Gamma(\alpha)}+\sum_{t<t_{i}} L_{i} t_{i}^{1-\alpha} \\
& \leq r, \\
& \left|D^{\alpha-1} \mathrm{Tu}(t)\right| \leq 2 \int_{0}^{\infty}\left|f\left(s, u(s), J^{\beta} u(s), D^{\alpha-1} u(s)\right)\right| \mathrm{d} s+\left|u_{0}\right|+\Gamma(\alpha) \sum_{t<t_{i}} L_{i} t_{i}^{1-\alpha} \\
& \leq 2\|u\|_{\mathrm{PC}^{1}} \int_{0}^{+\infty}\left[\left(1+s^{\alpha-1}\right) a(s)+b(s)+\frac{(1+s)^{\alpha-1} s^{\beta}}{\Gamma(\beta+1)} e(s)\right] \mathrm{d} s \\
& +2 \int_{0}^{+\infty} c(s) \mathrm{d} s+\left|u_{0}\right|+\Gamma(\alpha) \sum_{t<t_{i}} L_{i} t_{i}^{1-\alpha}
\end{aligned}
$$

$\leq r$. 
So, $\|\mathrm{Tu}\|_{\mathrm{PC}^{1}} \leq r$ and $T: B_{r} \longrightarrow B_{r}$.

Thirdly, we show that $\mathrm{TB}_{r}$ is uniformly bounded. From (38) and (39), we know

$$
\begin{array}{r}
\sup _{t \in J}\left|\frac{\mathrm{Tu}(t)}{1+t^{\alpha-1}}\right|<\infty, \\
\sup _{t \in J}\left|D^{\alpha-1} \mathrm{Tu}(t)\right|<\infty .
\end{array}
$$

So, for $u \in B_{r}$, it is easy to know that $\|\mathrm{Tu}\|_{\mathrm{PC}^{1}}<\infty$. Hence, $\mathrm{TB}_{r}$ is uniformly bounded.

Fourth, we prove that for any $u(t) \in B_{r},\left(\mathrm{Tu}(t) / 1+t^{\alpha-1}\right)$ and $D^{\alpha-1} \mathrm{Tu}(t)$ are equicontinuous on any interval $J_{k}$.

For any $u(t) \in B_{r}, t_{1}, t_{2} \in J_{k}(k=0,1,2, \ldots, m), t_{1}<t_{2}$, we have

$$
\begin{aligned}
\left|\frac{\mathrm{Tu}\left(t_{2}\right)}{1+t_{2}^{\alpha-1}}-\frac{\mathrm{Tu}\left(t_{1}\right)}{1+t_{1}^{\alpha-1}}\right| \leq & \frac{1}{\Gamma(\alpha)} \int_{0}^{t_{1}}\left|\frac{\left(t_{2}-s\right)^{\alpha-1}}{1+t_{2}^{\alpha-1}}-\frac{\left(t_{1}-s\right)^{\alpha-1}}{1+t_{1}^{\alpha-1}}\right| f\left(s, u(s), J^{\beta} u(s), D^{\alpha-1} u(s)\right) \mid \mathrm{d} s \\
& +\frac{1}{\Gamma(\alpha)} \int_{t_{1}}^{t_{2}} \frac{\left(t_{2}-s\right)^{\alpha-1}}{1+t_{2}^{\alpha-1}}\left|f\left(s, u(s), J^{\beta} u(s), D^{\alpha-1} u(s)\right)\right| \mathrm{d} s \\
& +\frac{1}{\Gamma(\alpha)} \int_{0}^{\infty}\left|f\left(s, u(s), J^{\beta} u(s), D^{\alpha-1} u(s)\right)\right| \mathrm{d} s\left|\frac{t_{2}^{\alpha-1}}{1+t_{2}^{\alpha-1}}-\frac{t_{1}^{\alpha-1}}{1+t_{1}^{\alpha-1}}\right| \\
& +\frac{\left|u_{0}\right|}{\Gamma(\alpha)}\left|\frac{t_{2}^{\alpha-1}}{1+t_{2}^{\alpha-1}}-\frac{t_{1}^{\alpha-1}}{1+t_{1}^{\alpha-1}}\right|+\frac{\sum_{t<t_{2}} L_{i} t_{i}^{1-\alpha}}{\Gamma(\alpha)}\left|\frac{t_{2}^{\alpha-1}}{1+t_{2}^{\alpha-1}}-\frac{t_{1}^{\alpha-1}}{1+t_{1}^{\alpha-1}}\right| \longrightarrow 0 \text { if } t_{2} \longrightarrow t_{1}, \\
\left|D^{\alpha-1} \mathrm{Tu}\left(t_{2}\right)-D^{\alpha-1} \mathrm{Tu}\left(t_{1}\right)\right| \leq & \int_{t_{1}}^{t_{2}}\left|f\left(s, u(s), J^{\beta} u(s), D^{\alpha-1} u(s)\right)\right| \mathrm{d} s+\left|\Gamma(\alpha) \sum_{t_{2}<t_{i}} I_{i} t_{i}^{1-\alpha}-\Gamma(\alpha) \sum_{t_{1}<t_{i}} I_{i} t_{i}^{1-\alpha}\right| \longrightarrow 0 \text { if } t_{2} \longrightarrow t_{1} .
\end{aligned}
$$

Therefore, for any $u(t) \in B_{r}, \quad \mathrm{Tu}(t) / 1+t^{\alpha-1}$ and $D^{\alpha-1} \mathrm{Tu}(t)$ are equicontinuous on any interval $J_{k}$.

Fifth, we need to verify that condition (3) in Lemma 2 is satisfied. It means that we need to verify $\mathrm{Tu}(t) / 1+t^{\alpha-1}$ and
$D^{\alpha-1} \mathrm{Tu}(t) \quad$ are equiconvergent at $t=J_{k}(k=1,2, \ldots, m, \ldots$,$) and t=\infty$ for any $u \in B_{r}$. We have

$$
\begin{aligned}
\lim _{t \rightarrow \infty} \frac{|\mathrm{Tu}(t)|}{1+t^{\alpha-1}} \leq & \lim _{t \rightarrow \infty}\left[\frac{2}{\Gamma(\alpha)} \int_{0}^{\infty}\left|f\left(s, u(s), J^{\beta} u(s), D^{\alpha-1} u(s)\right)\right| \mathrm{d} s \frac{t^{\alpha-1}}{1+t^{\alpha-1}}+\frac{\left|u_{0}\right|}{\Gamma(\alpha)} \frac{t^{\alpha-1}}{1+t^{\alpha-1}}+\sum_{t<t_{i}} L_{i} t_{i}^{1-\alpha} \frac{t^{\alpha-1}}{1+t^{\alpha-1}}\right] \\
\leq & \left(\frac{2\|u\|_{\mathrm{PC}}}{\Gamma(\alpha)} \int_{0}^{\infty}\left(\left(1+s^{\alpha-1}\right) a(s)+b(s)+\frac{(1+s)^{\alpha-1} s^{\beta}}{\Gamma(\beta+1)} e(s)\right) \mathrm{d} s+\frac{2}{\Gamma(\alpha)} \int_{0}^{\infty} c(s) \mathrm{d} s+\frac{u_{0}}{\Gamma(\alpha)}+\sum_{t<t_{i}} L_{i} t_{i}^{1-\alpha}\right) \\
& \lim _{t \rightarrow \infty} \frac{t^{\alpha-1}}{1+t^{\alpha-1}}<\infty, \\
\lim _{t \rightarrow \infty}\left|D^{\alpha-1} \mathrm{Tu}(t)\right| & <\lim _{t \rightarrow \infty}\left[\int_{0}^{\infty}\left|f\left(s, u(s), J^{\beta} u(s), D^{\alpha-1} u(s)\right)\right| \mathrm{d} s+\int_{0}^{\infty}\left|f\left(s, u(s), J^{\beta} u(s), D^{\alpha-1} u(s)\right)\right| \mathrm{d} s+\left|u_{0}\right|+\Gamma(\alpha) \sum_{t<t_{i}} L_{i} t_{i}^{1-\alpha}\right] \\
& <\lim _{t \rightarrow \infty}\left[2\|u\|_{P C^{1}} \int_{0}^{\infty}\left(\left(1+s^{\alpha-1}\right) a(s)+b(s)+\frac{(1+s)^{\alpha-1} s^{\beta}}{\Gamma(\beta+1)} e(s)\right) \mathrm{d} s+2 \int_{0}^{\infty} c(s) \mathrm{d} s+\left|u_{0}\right|+\Gamma(\alpha) \sum_{t<t_{i}} L_{i} t_{i}^{1-\alpha}\right] \\
& <\infty .
\end{aligned}
$$


Hence, $\mathrm{TB}_{r}$ is equiconvergent at infinity.

Then, we prove that $\mathrm{Tu}(t) / 1+t^{\alpha-1}$ and $D^{\alpha-1} \mathrm{Tu}(t)$ are equiconvergent at $t \longrightarrow t_{k}^{+}(k=0,1,2, \ldots$,$) . We have$

$$
\begin{aligned}
& \lim _{t \longrightarrow t_{k}^{+}} \mid \frac{\mathrm{Tu}(t)}{1+t_{k}^{\alpha-1}}+\frac{1}{\Gamma(\alpha)} \int_{0}^{t_{k}} \frac{\left(t_{k}-s\right)^{\alpha-1}}{1+t_{k}^{\alpha-1}} f\left(s, u(s), J^{\beta} u(s), D^{\alpha-1} u(s)\right) \mathrm{d} s-\frac{t_{k}^{\alpha-1}}{\Gamma(\alpha)\left(1+t_{k}^{\alpha-1}\right)} \\
& \int_{0}^{\infty} f\left(s, u(s), J^{\beta} u(s), D^{\alpha-1} u(s)\right) \mathrm{d} s \\
& -\frac{t_{k}^{\alpha-1}}{\Gamma(\alpha)\left(1+t_{k}^{\alpha-1}\right)} u_{0}+\frac{t_{k}^{\alpha-1}}{1+t_{k}^{\alpha-1}} \sum_{t_{k}<t_{i}} I_{i} t_{i}^{1-\alpha} \mid=0, \\
& \lim _{t \longrightarrow t_{k}^{+}}\left|D^{\alpha-1} \mathrm{Tu}(t)+\int_{0}^{t_{k}} f\left(s, u(s), J^{\beta} u(s), D^{\alpha-1} u(s)\right) \mathrm{d} s-\int_{0}^{\infty} f\left(s, u(s), J^{\beta} u(s), D^{\alpha-1} u(s)\right) \mathrm{d} s-u_{0}+\Gamma(\alpha) \sum \sum_{t_{k}<t_{i}} I_{i}^{1-\alpha}\right|=0 .
\end{aligned}
$$

Therefore, $\mathrm{Tu}(t) / 1+t^{\alpha-1}$ and $D^{\alpha-1} \mathrm{Tu}(t)$ are equiconvergent at $t=J_{k}(k=1,2, \ldots, m, \ldots$,$) and t=\infty$ for any $u \in B_{r}$. By using Lemma 2 , we obtain that $\mathrm{TB}_{r}$ is relatively compact, that is, $T$ is a compact operator.

Therefore, Schauder's fixed-point theorem implies that problem (1) has at least one solution in $B_{r}$.
Our second result is based on Altman fixed-point theorem.

Theorem 4. Assume (H2) and the following condition hold:

(H3) For $f \in C([0,+\infty) \times R \times R \times R, R)$, there exist nonnegative functions $a(t), b(t), c(t)$ defined on $[0, \infty)$ and constants $p, q, l \geq 0$ such that

$$
\begin{aligned}
|f(t, x, y, z)| \leq a(t)+b(t)|x|^{p}+c(t)|y|^{q}+e(t)|z|^{l} \\
\int_{0}^{+\infty} a(t) \mathrm{d} t=a^{*}<\infty, \int_{0}^{+\infty}\left(1+t^{\alpha-1}\right)^{p} b(t) \mathrm{d} t=b^{*}<+\infty, \int_{0}^{+\infty} c(t) \mathrm{d} t=c^{*}<+\infty \\
\\
\quad \int_{0}^{+\infty}\left(\frac{(1+t)^{\alpha-1} t^{\beta}}{\Gamma(\beta+1)}\right)^{l} e(t) \mathrm{d} t=e^{*}<\infty
\end{aligned}
$$

If $0 \leq p, q, l<1$, then problem (1) has at least one solution Proof. Let us choose $u(t)$ in $P C^{1}(J, \mathbb{R})$.

$$
R \geq \max \left\{12 a^{*},\left(12 b^{*}\right)^{1 / 1-p},\left(12 c^{*}\right)^{1 / 1-q},\left(12 e^{*}\right)^{1 / 1-l}, 6\left|u_{0}\right|, 6 \Gamma(\alpha) \sum_{t<t_{i}} L_{i} t_{i}^{1-\alpha}\right\}
$$


and define $U=\left\{u \in \mathrm{PC}^{1}\|u\|_{\mathrm{PC}^{1}} \leq R\right\}$. According to Theorem 3 , we know $T: U \longrightarrow U$ is a completely continuous operator. For any $u \in \partial U$, by (H3), we have

$$
\begin{aligned}
& \frac{\mathrm{Tu}(t)}{1+t^{\alpha-1}} \leq \frac{2}{\Gamma(\alpha)} \int_{0}^{\infty}\left|f\left(s, u(s), J^{\beta} u(s), D^{\alpha-1} u(s)\right)\right| \mathrm{d} s+\frac{\left|u_{0}\right|}{\Gamma(\alpha)}+\sum_{t<t_{i}} L_{i} t_{i}^{1-\alpha} \\
& \leq \frac{2}{\Gamma(\alpha)}\left(\int_{0}^{\infty}\left[a(s)+b(s)|u(s)|^{p}+c(s)\left|D^{\alpha-1} u(s)\right|^{q}+e(s)\left|J^{\beta} u(s)\right|^{l}\right] \mathrm{d} s\right)+\frac{\left|u_{0}\right|}{\Gamma(\alpha)}+\sum_{t<t_{i}} L_{i} t_{i}^{1-\alpha} \\
& \leq \frac{2}{\Gamma(\alpha)}\left(a^{*}+\int_{0}^{\infty} b(s)\left(1+s^{\alpha-1}\right)^{p} \frac{|u(s)|^{p}}{\left(1+s^{\alpha-1}\right)^{p}} \mathrm{~d} s+\int_{0}^{\infty} c(s)\|u\|_{\mathrm{PC}^{1}}^{q} \mathrm{~d} s+\int_{0}^{+\infty} e(s)\left(\frac{(1+s)^{\alpha-1} s^{\beta}}{\Gamma(\beta+1)}\right)^{l}\|u\|_{\mathrm{PC}^{1}}^{l} \mathrm{~d} s\right) \\
&+\frac{\left|u_{0}\right|}{\Gamma(\alpha)}+\sum_{t<t_{i}} L_{i} t_{i}^{1-\alpha} \\
& \leq \frac{2}{\Gamma(\alpha)}\left(a^{*}+b^{*}\|u\|_{\mathrm{PC}^{1}}^{p}+c^{*}\|u\|_{\mathrm{PC}^{1}}^{q}+e^{*}\|u\|_{\mathrm{PC}}^{l}\right)+\frac{\left|u_{0}\right|}{\Gamma(\alpha)}+\sum_{t<t_{i}} L_{i} t_{i}^{1-\alpha} \\
& \leq \frac{2}{\Gamma(\alpha)}\left(\frac{R}{12}+\frac{R}{12}+\frac{R}{12}+\frac{R}{12}\right)+\frac{R}{6 \Gamma(\alpha)}+\frac{R}{6 \Gamma(\alpha)} \\
&<R, \\
& \leq \leq 2\left(\frac{R}{12}+\frac{R}{12}+\frac{R}{12}+\frac{R}{12}\right)+\frac{R}{6}+\frac{R}{6}=R . \\
&\left|D^{\alpha-1} \mathrm{Tu}(t)\right| \leq 2 \int_{0}^{\infty}\left|f\left(s, u(s), J^{\beta} u(s), D^{\alpha-1} u(s)\right)\right| \mathrm{d} s+\left|u_{0}\right|+\Gamma(\alpha) \sum_{t<t_{i}} L_{i} t_{i}^{1-\alpha} \\
& \\
&\left.\leq b^{*}\|u\|_{\mathrm{PC}^{1}}^{p}+c^{*}\|u\|_{\mathrm{PC}^{1}}^{q}+e^{*}\|u\|_{\mathrm{PC}}^{l}\right)+\left|u_{0}\right|+\Gamma(\alpha) \sum_{t<t_{i}} L_{i} t_{i}^{1-\alpha}
\end{aligned}
$$

Thus, from (49) and (50), we have $\mathrm{TU} \subset U$ and $\|\mathrm{Tu}\|_{\mathrm{PC}^{1}} \leq\|u\|_{\mathrm{PC}^{1}}, \forall u \in \partial U$. So, by Theorem 2, we know that problem (1) has at least one solution.

Theorem 5. Assume that conditions $(\mathrm{H} 2)$ and $(\mathrm{H} 3)$ are satisfied. If $p=q=l=1,(1+\Gamma(\alpha))\left(b^{*}+c^{*}\right)<\Gamma(\alpha)$, then problem (1) has at least one solution.
Proof. Let us take

$$
R>\frac{\left|u_{0}\right|+\Gamma(\alpha) \sum_{t<t_{i}} L_{i} t_{i}^{1-\alpha}+2 a^{*}}{1-2\left(b^{*}+c^{*}+e^{*}\right)},
$$

and define $U=\left\{u \in \mathrm{PC}^{1} \mid\|u\|_{\mathrm{PC}^{1}}<R\right\}$.

For any $u \in \partial U$, we have 


$$
\begin{aligned}
\frac{\mathrm{Tu}(t)}{1+t^{\alpha-1}} & \leq \frac{2}{\Gamma(\alpha)} \int_{0}^{\infty}\left|f\left(s, u(s), J^{\beta} u(s), D^{\alpha-1} u(s)\right)\right| \mathrm{d} s+\frac{\left|u_{0}\right|}{\Gamma(\alpha)}+\sum_{t<t_{i}} L_{i} t_{i}^{1-\alpha} \\
& \leq \frac{2}{\Gamma(\alpha)}\left(\int_{0}^{\infty}\left[a(s)+b(s)|u(s)|+c(s)\left|D^{\alpha-1} u(s)\right|+e(s)\left|J^{\beta} u(s)\right|\right] \mathrm{d} s\right)+\frac{\left|u_{0}\right|}{\Gamma(\alpha)}+\sum_{t<t_{i}} L_{i} t_{i}^{1-\alpha} \\
& \leq \frac{2}{\Gamma(\alpha)} a^{*}+\left[\int_{0}^{\infty} b(s)\left(1+s^{\alpha-1}\right) \frac{|u(s)|}{\left(1+s^{\alpha-1}\right)} \mathrm{d} s+\int_{0}^{\infty} c(s)\|u\|_{\mathrm{PC}^{1}} \mathrm{~d} s+\int_{0}^{\infty} e(s) \frac{|u(s)|}{\left(1+s^{\alpha-1}\right)} \frac{(1+s)^{\alpha-1} s^{\beta}}{\Gamma(\beta+1)}\right]+\frac{\left|u_{0}\right|}{\Gamma(\alpha)}+\sum_{t<t_{i}} L_{i} t_{i}^{1-\alpha} \\
& \leq \frac{2}{\Gamma(\alpha)}\left(a^{*}+b^{*}\|u\|_{\mathrm{PC}^{1}}+c^{*}\|u\|_{\mathrm{PC}^{1}}+e^{*}\|u\|_{\mathrm{PC}^{1}}\right)+\frac{\left|u_{0}\right|}{\Gamma(\alpha)}+\sum_{t<t_{i}} L_{i} t_{i}^{1-\alpha} \\
& \leq \frac{2}{\Gamma(\alpha)}\left(a^{*}+b^{*} R+c^{*} R+e^{*} R\right)+\frac{\left|u_{0}\right|}{\Gamma(\alpha)}+\sum_{t<t_{i}} L_{i} t_{i}^{1-\alpha} \\
& <R
\end{aligned}
$$$$
\left|D^{\alpha-1} \mathrm{Tu}(t)\right| \leq 2 \int_{0}^{\infty}\left|f\left(s, u(s), J^{\beta} u(s), D^{\alpha-1} u(s)\right)\right| \mathrm{d} s+\left|u_{0}\right|+\Gamma(\alpha) \sum_{t<t_{i}} L_{i} t_{i}^{1-\alpha}
$$$$
\leq 2\left(a^{*}+b^{*}\|u\|_{\mathrm{PC}^{1}}+c^{*}\|u\|_{\mathrm{PC}^{1}}+e^{*}\|u\|_{\mathrm{PC}^{1}}\right)+\left|u_{0}\right|+\Gamma(\alpha) \sum_{t<t_{i}} L_{i} t_{i}^{1-\alpha}
$$$$
\leq 2\left(a^{*}+b^{*} R+c^{*} R+e^{*} R\right)+\left|u_{0}\right|+\Gamma(\alpha) \sum_{t<t_{i}} L_{i} t_{i}^{1-\alpha}
$$$$
<R
$$

Thus, from (52) and (53), we have TU $\subset U$ and $\|\mathrm{Tu}\|_{\mathrm{PC}^{1}} \leq\|u\|_{\mathrm{PC}^{1}}, \forall u \in \partial U$. So, by Theorem 2, we know that problem (1) has at least one solution.

Remark 1. If we use other conditions instead of the condition " $p=q=1$ ", for example, $0 \leq p<1, q=1$ or $p>1, q=$ 1 or $0 \leq q<1, p=1$ or $q>1, p=1$ or $p, q>1$ or $0 \leq p<1, q>1$ or $0 \leq q<1, p>1$, and choose proper $R$, respectively, then we can obtain the same result. The proof is similar to Theorem 4 or Theorem 5, so we omit it.

\section{Example}

In this section, we give an example to illustrate of our main result.

Example 1. Consider the following impulsive boundary value problem of fractional order:

$$
\left\{\begin{array}{l}
D_{0+}^{3 / 2} u(t)+\frac{\ln \left(\left(1+\left|D_{0+}^{1 / 2} u(t)\right|\right)\right.}{20\left(1+t^{2}\right)}+\frac{\sqrt{\left|u(t) D_{0+}^{1 / 2} u(t)\right|}}{20 e^{\sqrt{t}}}+\frac{\left|J^{3 / 2} u(t)\right|}{20 e^{t}}=0, \quad t \in[0, \infty) \backslash\left\{\frac{1}{2}\right\}, \\
\triangle u\left(\frac{1}{2}\right)=I\left(u\left(\frac{1}{2}\right)\right), \quad t=\frac{1}{2}, \\
u(0)=u^{\prime}(0)=0, \quad D_{0+}^{1 / 2} u(\infty)=u_{0},
\end{array}\right.
$$


where $\quad \alpha=3 / 2, \quad f(t, x, y, z)=\ln (1+|y|) / 20\left(1+t^{2}\right)+$ $\sqrt{|x y|} / 20 e^{\sqrt{t}}+|z| / 20 e^{t}, k=1, t_{1}=1 / 2$.

Let $I(u)=1 /(u+1 / u)$. Then, we have

$$
\begin{aligned}
|f(t, x, y)| & \leq \frac{1}{40 e^{\sqrt{t}}}|x|+\left(\frac{1}{20\left(1+t^{2}\right)}+\frac{1}{40 e^{\sqrt{t}}}\right)|y|+\frac{1}{20 e^{t}}|z|, \\
I(u) & =\frac{1}{|u|+1 /|u|} \leq 1 .
\end{aligned}
$$

By computing, we know that

$$
\begin{gathered}
\int_{0}^{+\infty}\left[\left(1+t^{\alpha-1}\right) a(t)+b(t)\right] \mathrm{d} t=\int_{0}^{+\infty}\left[\left(1+t^{1 / 2}\right) \frac{1}{40 e^{\sqrt{t}}}+\frac{1}{20\left(1+t^{2}\right)}+\frac{1}{40 e^{\sqrt{t}}}\right] \mathrm{d} t=\frac{1}{5}+\frac{\pi}{40} \approx 0.2785<\infty, \\
\int_{0}^{+\infty} \frac{(1+t)^{\alpha-1} t^{\beta}}{\Gamma(\beta+1)} e(t) \mathrm{d} t=\int_{0}^{+\infty} \frac{(1+t)^{1 / 2} t^{3 / 2}}{\Gamma(5 / 2)} \frac{1}{20 e^{t}} \approx 64.5850<\infty .
\end{gathered}
$$

Thus, the conditions of Theorem 3 are satisfied, and hence problem (54) has at least one solution.

Remark 2. By theorems in $[9,10,13]$, this problem could not be solved.

\section{Data Availability}

No data were used to support this study.

\section{Conflicts of Interest}

The authors declare that they have no conflicts of interest.

\section{References}

[1] M. Benchonra and B. A. Slimani, "Existence and uniqueness of solutions to impulsive fractional differential equations," Electronic Journal of Differential Equations, vol. 2009, no. 10, pp. 1-11, 2009.

[2] X. Su and S. Zhang, "Unbounded solutions to a boundary value problem of fractional order on the half-line," Computers \& Mathematics with Applications, vol. 61, no. 4, pp. 10791087, 2011.

[3] C. Shen, H. Zhou, and L. Yang, "On the existence of solution to a boundary value problem of fractional differential equation on the infinite interval," Boundary Value Problems, vol. 325, pp. 1-13, 2015.

[4] Y. Wang, Y. Liu, and Y. Cui, "Infinitely many solutions for impulsive fractional boundary value problem with p-Laplacian,” Boundary Value Problems, vol. 2018, p. 94, 2018.

[5] Y. Liu, "Bifurcation techniques for a class of boundary value problems of fractional impulsive differential equations," Journal of Nonlinear Sciences and Applications, vol. 9, no. 4, pp. 340-353, 2015.

[6] Nl. Mahmudov and S. Unul, "On existence of BVP's for impulsive fractional differential equations," Advances in Difference Equations, vol. 15, pp. 1-16, 2017.

[7] Y. Hu and F. Li, "Existence of solutions for the nonlinear multiple base points impulsive fractional differential equations with the three-point boundary conditions," Advances in Difference Equations, vol. 55, pp. 1-14, 2017.

[8] K. Zhao and J. Liang, "Solvability of triple-point integral boundary value problems for a class of impulsive fractional differential equations," Advances in Difference Equations, vol. 50, pp. 1-19, 2017.

[9] X. Zhao and W. Ge, "Some results for fractional impulsive boundary value problems on infinite intervals," Applications of Mathematics, vol. 56, no. 4, pp. 371-387, 2011.

[10] Y. Liu and B. Ahmad, "A study of impulsive multiterm fractional differential equations with single and multiple base points and applications," The Scientific World Journal, vol. 2014, pp. 1-28, Article ID 194346, 2014.

[11] L. Yang and H. Chen, "Nonlocal boundary value problem for impulsive differential equations of fractional order," Advances in Difference Equations, vol. 2011, pp. 1-16, Article ID 404917, 2011.

[12] X. Fu and X. Bao, "Some existence results for nonlinear fractional differential equations with impulsive and fractional integral boundary conditions," Advances in Difference Equations, vol. 129, pp. 1-10, 2014.

[13] Y. Liu, "On piecewise continuous solutions of higher order impulsive fractional differential equations and applications," Applied Mathematics and Computation, vol. 287-288, pp. 38-49, 2016. 